\title{
Cell-Type-Specific Epigenetic Editing at the Fosb Gene Controls Susceptibility to Social Defeat Stress
}

\author{
Peter J Hamilton', Dominika J Burek', Sonia I Lombroso², Rachael L Neve ${ }^{3}$, Alfred J Robison ${ }^{4}$, Eric J Nestler' \\ and Elizabeth A Heller*,2 \\ 'Fishberg Department of Neuroscience and Friedman Brain Institute, Icahn School of Medicine at Mount Sinai, New York, NY, USA; ${ }^{2}$ Department \\ of Systems Pharmacology and Translational Therapeutics, Perelman School of Medicine, University of Pennsylvania, Philadelphia, PA, USA; \\ ${ }^{3}$ McGovern Institute for Brain Research, Massachusetts Institute of Technology, Cambridge, MA, USA; ${ }^{4}$ Department of Physiology, Michigan State \\ University, East Lansing, MI, USA
}

\begin{abstract}
Chronic social defeat stress regulates the expression of Fosb in the nucleus accumbens (NAc) to promote the cell-type-specific accumulation of $\triangle$ FosB in the two medium spiny neuron (MSN) subtypes in this region. $\triangle$ FosB is selectively induced in DI-MSNs in the NAc of resilient mice, and in D2-MSNs of susceptible mice. However, little is known about the consequences of such selective induction, particularly in D2-MSNs. This study examined how cell-type-specific control of the endogenous Fosb gene in NAc regulates susceptibility to social defeat stress. Histone post-translational modifications (HPTMs) were targeted specifically to Fosb using engineered zinc-finger proteins (ZFPs). Fosb-ZFPs were fused to either the transcriptional repressor, G9a, which promotes histone methylation or the transcriptional activator, p65, which promotes histone acetylation. These ZFPs were expressed in DI-vs D2-MSNs using Cre-dependent viral expression in the NAc of mice transgenic for Cre recombinase in these MSN subtypes. We found that stress susceptibility is oppositely regulated by the specific cell type and HPTM targeted. We report that Fosb-targeted histone acetylation in D2-MSNs or histone methylation in DI-MSNs promotes a stress-susceptible, depressive-like phenotype, while histone methylation in D2-MSNs or histone acetylation in DI-MSNs increases resilience to social stress as quantified by social interaction behavior and sucrose preference. This work presents the first demonstration of cell- and gene-specific targeting of histone modifications, which model naturally occurring transcriptional phenomena that control social defeat stress behavior. This epigenetic-editing approach, which recapitulates physiological changes in gene expression, reveals clear differences in the social defeat phenotype induced by Fosb gene manipulation in MSN subtypes.
\end{abstract}

Neuropsychopharmacology (2018) 43, 272-284; doi:I0.1038/npp.20 17.88; published online 7 June 2017

\section{INTRODUCTION}

While acute exposure to stressful or rewarding stimuli transiently induces many Fos family transcription factors in the nucleus accumbens (NAc), chronic stimulation causes the stable accumulation of $\triangle \mathrm{FosB}$, a truncated product of the FosB gene (Hope et al, 1994), which mediates the bidirectional regulation of hundreds of $\Delta$ FosB-target genes in this brain region (McClung and Nestler, 2003; Robison and Nestler, 2011). Studies to elucidate the mechanism of Fosb activation by chronic stimuli have uncovered a crucial role of epigenetic remodeling. Drug and stress exposure regulate histone post-translational modifications (HPTMs) and transcription factor binding at the Fosb locus to control its expression (Maze et al, 2010; Vialou et al, 2010a). Recently, we demonstrated that targeting HPTMs specifically to Fosb

* Correspondence: Dr EA Heller, Department of Systems Pharmacology and Translational Therapeutics, Perelman School of Medicine, University of Pennsylvania, Philadelphia, PA 19106, USA, Tel: 2I5 573 7038, Fax: 215573 9135, E-mail: eheller@mail.med.upenn.edu

Received 15 February 2017; revised 12 April 2017; accepted 25 April 2017; accepted article preview online 2 May 2017 in NAc neurons was sufficient to bidirectionally regulate Fosb gene expression as well as drug- and stress-evoked behaviors (Heller et al, 2014).

The NAc is composed mainly ( 95\%) of GABAergic projection medium spiny neurons (MSNs), which are segregated into two subtypes based on their specific enrichment of many genes, including dopamine receptor D1 (Drd1) or dopamine receptor D2 (Drd2), and by their differential outputs to subcortical structures (Graybiel, 2000; Kupchik et al, 2015; Lobo et al, 2006). Studies have revealed a role of $\Delta$ FosB accumulation in D1-type MSNs in mediating rewarding behaviors. $\Delta \mathrm{FosB}$ induction in response to chronic administration of drugs of abuse predominates in D1-MSNs (Lobo et al, 2013), and its overexpression in this cell type causes plasticity at glutamatergic synapses in NAc (Grueter et al, 2013; Robison et al, 2013) as well as increased locomotor responses to cocaine (Kelz et al, 1999), increased conditioned place preference to both cocaine and morphine (Zachariou et al, 2006), and enhanced cocaine selfadministration (Colby et al, 2003).

Cell-type-specific Fosb regulation also appears to be a critical mediator of depressive disorders, given that $\Delta \mathrm{FosB}$ 
expression is reduced (Vialou et al, 2010b), and repressive histone methylation is increased at Fosb in the NAc of human depressed patients (Heller et al, 2014). The chronic social defeat stress (CSDS) model relies on territorial aggression, a natural mouse behavior, to produce core symptoms of depression as assessed by deficits in social interaction and reward preference (Donahue et al, 2014; Krishnan et al, 2007). Recently, Lobo et al (2013) found that $\triangle$ FosB expression is increased in the NAc after CSDS specifically in D2-MSNs of susceptible mice and in D1-MSNs of resilient mice. While $\Delta$ FosB overexpression in D1-MSNs of NAc promotes resilience to CSDS (Vialou et al, 2010b), the consequences of its induction in D2-MSNs remains unknown, and the reliance on overexpression systems may produce non-physiological results. Moreover, the causal relationship between stress-related behaviors and alterations in HPTMs at a single gene locus within these specific MSN subtypes remains unexplored. We have recently reported that Fosb-targeted histone $\mathrm{H} 3$ lysine 9 dimethylation (H3K9me2) in all NAc neurons promotes stress susceptibility (Heller et al, 2014), yet studies have found opposing functional roles of the G9a-mediated repressive histone modification, $\mathrm{H} 3 \mathrm{~K} 9 \mathrm{me} 2$, in D1- and D2-MSNs (Maze et al, 2014; Schaefer et al, 2009). We therefore sought to uncover the cell-type-specific function of HPTM-mediated repression of Fosb in responses to CSDS.

To examine the role of epigenetic remodeling at the Fosb gene specifically in D1 and D2 NAc MSNs, we injected Cre-dependent viral vectors into the NAc of bacterial artificial chromosome (BAC) transgenic mice that selectively express Cre recombinase in either of these cell types. While previous studies have demonstrated that $\mathrm{Fos} B / \Delta \mathrm{FosB}$ protein expression is differentially regulated in the two MSN cell types, the basal level of FosB/ $\Delta$ FosB protein in the D1 and D2-Cre transgenic lines is the same (Lobo et al, 2013). Furthermore, while there is evidence that a subset of NAc MSNs express both D1 and D2 receptor proteins (Hasbi et al, 2009; Shetreat et al, 1996; Surmeier et al, 1996; Yung et al, 1995), extensive characterization of the D1 and D2-Cre transgenic mice used in this study find virtually no overlap of the Cre-expressing populations (Bateup et al, 2008; Lobo et $a l, 2013)$. We targeted HPTMs specifically to the Fosb promoter using engineered zinc-finger proteins (ZFPs), fused to either the transcriptional repressor, G9a, or transcriptional activator, p65 (Heller et al, 2014). We cloned our ZFP constructs into plasmids containing a lox-stop-lox cassette (LS1L) and packaged these plasmids into herpes simplex virus (HSV). We then delivered the HSVs into the NAc of BAC transgenic mice that selectively express Cre recombinase in either D1- or D2-MSNs, thus allowing for cell-type-specific expression of our ZFPs and, in turn, celltype-specific epigenetic editing. Using this approach we found that epigenetic remodeling of the Fosb gene has opposite roles in these two NAc MSN subtypes in mediating the depressive-like phenotype evoked by social defeat stress.

\section{MATERIALS AND METHODS}

\section{Animals and Treatments}

Adult male 7-8-week-old mice and 6-month-old CD1 retired breeders (CD1 aggressors) were housed at $22-25^{\circ} \mathrm{C}$ in a $12-\mathrm{h}$ light-dark cycle and provided food and water ad libitum. All tests were conducted during the light cycle. Members of the same cage were randomly assigned to different experimental groups for behavioral studies. Experiments were conducted in accordance with the guidelines of the Institutional Animal Care and Use Committee at Mount Sinai.

\section{Transcription Factor Engineering}

All ZFPs were manufactured by the CompoZr ZFN Operations Group at Sigma-Aldrich Biotechnology and cloned in frame N-terminal to the p65 activation (Liu et al, 2001), or G9a repression domain (pre-SET and SET domains only) (Snowden et al, 2002). The Fosb-ZFP recognizes a 19 bp motif in the FosB promoter (5'-GATCCCCTCCCGCGAAGCC-3'), which is unique to the mouse genome (Heller et al, 2014).

\section{Viral-Mediated Gene Transfer}

Fosb-ZFPs were cloned into bicistronic herpes simplex viral vector (HSV-LS1L-Fosb-ZFP-p65/G9a) (Grueter et al, 2013), which expresses GFP under the CMV promoter. Fosb-ZFPs were inserted following a stop codon surrounded by loxP sites driven by the IE 4/5 promoter allowing expression of GFP in all transduced cells and expression of Fosb-ZFPs only in cells also expressing Cre recombinase. For visualization of Cre+ cells, mCherry was cloned into HSV vector with a Cre-dependent transcription cassette (HSV-LS1L-mCherry). Expression of the HSV transgene is maximal by 3-5 days after infusion and dissipates by days 8-10. Social defeat experiments were thus designed within this time frame. HSV vectors were selected because, unlike other types of viral vectors, HSVs have sufficient capacity to package the large ZFP constructs $(>4.5 \mathrm{~kb})$ and specifically infect neurons in vitro and in vivo (Neve et al, 2005). There has been extensive prior characterization of HSV-mediated transduction of the NAc, and it has been observed that the vast majority of infected neurons in the NAc ( 95\%) are MSNs (Barrot et al, 2002).

\section{Stereotaxic Surgery}

Mice were anesthetized with a mixture of ketamine $(100 \mathrm{mg} /$ $\mathrm{kg})$ and xylazine $(10 \mathrm{mg} / \mathrm{kg})$ and positioned in a small animal stereotaxic instrument. NAc was targeted bilaterally using the following stereotaxic coordinates: +1.6 (anterior/posterior), +1.5 (medial/lateral), and -4.4 (dorsal/ventral) at an angle of $10^{\circ}$ from the midline (relative to Bregma). A total of $1 \mu \mathrm{l}$ of virus was delivered on each side over a 5 -min period, followed by $7 \mathrm{~min}$ of rest. In all behavioral experiments, proper NAc targeting of virus infusion was confirmed post hoc by preparing brain slices and visual confirmation of both needle track and GFP expression by stereoscopic fluorescence microscopy. Depending on the defeat paradigm (either accelerated or subthreshold), mice were allowed to recover for either 3 or 6 days post surgery, to standardize the amount of time between surgery and social interaction testing.

\section{Immunohistochemistry}

Mice were injected intra-NAc with an HSV cocktail containing a mixture of HSV-LS1L-Fosb-ZFP-G9a and 
HSV-LS1L-mCherry according to the above protocol. Brain tissue was cleared via transcardial perfusion of chilled PBS for $30 \mathrm{~s}$, followed by $5 \mathrm{~min}$ of $4 \%$ paraformaldehyde (PFA). Brains were postfixed overnight at $4{ }^{\circ} \mathrm{C}$ in $4 \%$ PFA. Immunofluorescence was performed using a mouse antiFosB (ab11959, 1:500; Abcam), a rat anti-mCherry (M11217, 1:1000; Invitrogen), and a goat anti-GFP (ab5450, 1:1000; Abcam) primary antibody and corresponding secondaries (705-545-147, 711-175-152, and 712-165-150, 1:200, Jackson Immunoresearch Labs). Fluorescent images were visualized on an Olympus FluoView 1000 Filter-based Laser Scanning Confocal Microscope. Resulting images were analyzed using the ImageJ software (NIH) for pixel intensity of the Cy5 channel (FosB staining) in the nucleus of cells selected by a double-blinded experimenter.

\section{Social Defeat and Behavior Testing}

Two social defeat paradigms were used to calibrate the effects of the social defeat and reveal shifts in susceptibility or resilience of mouse cohorts. For the accelerated social defeat, mice underwent 4 days of social defeat stress as described previously (Berton et al, 2006; Dias et al, 2014; Krishnan et al, 2007). Mice were exposed for four consecutive days to a novel aggressive CD1 retired breeder for $10 \mathrm{~min}$ in a large hamster cage two times daily (AM and PM). Between bouts, mice were housed separated from the aggressor by a perforated divider to maintain sensory contact. Alternatively, subthreshold social defeat was used to detect mechanisms that promote a susceptibility to social stress (Chaudhury et al, 2013; Krishnan et al, 2007). Mice were exposed to a novel CD1 male aggressor three times for $5 \mathrm{~min}$ each, with 15 min intervals between each exposure. Mice were tested for social interaction $24 \mathrm{~h}$ after the last social defeat interaction according to published protocols (Berton et al, 2006; Krishnan et al, 2007). Based on the interaction ratio, defined as time spent with target/time spent with no target, mice were characterized as susceptible $(<1)$ or resilient $(>1)$ (Krishnan et al, 2007). Mice were tested in a standard elevated plus maze (EPM) for $10 \mathrm{~min}$, monitored by Ethovision XT. Note that as with previously published protocols (Chaudhury et al, 2013; Heller et al, 2014; Heller et al, 2016; Walsh et al, 2014), this paradigm does not include a home-cage (non-defeated) control, as comparisons being made are within stressed groups.

\section{Sucrose Preference Test}

Immediately following the social interaction and EPM tests, mice were individually housed and given both a bottle containing water and a bottle containing a $1 \%$ sucrose solution with the left/right location balanced across animals. No previous food or water deprivation was applied before the test. Water and sucrose solution intake was measured daily by weighing the bottles. The positions of two bottles were exchanged every $24 \mathrm{~h}$, to avoid position preferences. After 3 days of habituation, the sucrose preference on the fourth day was expressed as ( $\Delta$ weight $\left._{\text {sucrose }}\right) /$ $\left(\Delta\right.$ weight $_{\text {sucrose }}+\Delta$ weight $\left._{\text {water }}\right) \times 100$.

\section{Statistics}

The appropriate statistical test was determined based on the number of comparisons being carried out. Student's $t$-tests were used for comparison of two groups, in the analysis of social interaction data, sucrose preference, and EPM. Effects were considered significant at $p<0.05$. Data are expressed as mean \pm SEM. All experiments were carried out one to three times, and data replication was observed in instances of repeated experiments.

\section{RESULTS \\ In vivo Validation of Cre-Dependent Expression of Engineered Transcription Factors}

Our group has previously reported the development and validation of engineered transcription factors in their ability to modify chromatin states proximal to targeted, highaffinity DNA binding sites (Heller et al, 2014, 2016). Specifically, we use engineered ZFPs targeted to the Fosb promoter and fused to either the transcriptional repressor, G9a, which catalyzes the deposition of $\mathrm{H} 3 \mathrm{~K} 9 \mathrm{me} 2$, or the transcriptional activator, $\mathrm{p} 65$, which mediates the enrichment of H3K9/14Ac at the Fosb locus. We have demonstrated that these epigenetic modifications, when delivered globally to neurons in the NAc, are sufficient to alter addiction- and depression-related behaviors of mice (Heller

Figure I Cre-dependent Fosb-ZFP viral vectors target Cre+ neurons in vivo. Wild-type (WT) $(n=5)$, DI-Cre $(n=3)$, and D2-Cre $(n=3)$ mice were injected intra nucleus accumbens (NAc) with a herpes simplex virus (HSV) mixture of HSV-LSI L-Fosb-ZFP-G9a and HSV-LSI L-mCherry (to visualize Cre+ cells) and were allowed to recover for 3 days. Brain tissue was cleared, formaldehyde crosslinked, and immunohistochemistry was performed. (a) Vector map cartoons of the HSV-LSI L-Fosb-ZFP-p65 and HSV-LSI L-Fosb-ZFP-G9a used in these studies. (b) Green fluorescence indicates successful transduction of the HSV-LSIL-Fosb-ZFP-G9a vector in WT, DI-Cre, and D2-Cre animals. Green fluorescent protein (GFP) is expressed under the control of a separate, non-Cre-dependent CMV promoter on the Fosb-ZFP expression vector (first column). mCherry indicates the presence of Cre+ neurons in DI- and D2-Cre mice, but not WT mice (second column). FosB expression levels in both Cre+ and Cre - cells are represented in the third column, with the merged channels shown in the fourth column. Scale bar = 10 $\mathrm{\mu m}$. (c) Among all virally infected neurons, FosB expression is altered only in Cre+ cells. FosB expression is not altered in Cre - cells in DI- and D2-Cre mice, or in WT mice, indicating that Fosb-ZFP-G9a functions only in Cre+ cells $(\mathrm{WT}, n=2 \mathrm{I}$; DI-Cre+, $n=22$; $\mathrm{D}$ I-Cre-, $n=16$; D2-Cre+, $n=14$; D2-Cre- $n=19$. * $p<0.05$; one-way analysis if variance (ANOVA) followed by Tukey's multiple comparison test: WT vs DI Cre+: mean diff., 0.4352, 95\% confidence interval (Cl) of diff., 0.09659-0.7738, *p<0.05; WT vs DI Cre - : mean diff., $0.1800,95 \%$ Cl of diff., -0.1883 to 0.5483 , NS; WT vs D2 Cre+: mean diff., $0.4644,95 \%$ Cl of diff., $0.08150-0.8474$, * p < 0.05 ; WT vs D2 Cre - : mean diff., $-0.01034,95 \%$ Cl of diff., -0.3618 to $0.34 \mathrm{II}$, NS). (d) There is no significant difference in FosB expression in non-infected cells in WT, DI-Cre, or D2-Cre mice (WT, $n=89$; DI-Cre, $n=9$; : D2-Cre, $n=68$. Not significant by one-way ANOVA followed by Tukey's multiple comparison test: WT vs DI Cre: mean diff., $0.2129,95 \%$ Cl of diff., -0.03077 to 0.4565 , NS; WT vs D2 Cre: mean diff., $-0.05225,95 \%$ Cl of diff., -0.3 I 55 to 0.2 I I 0, NS). NS, not significant. 
et al, 2014). In this study, we aimed to deliver these ZFPs to specific neuronal subpopulations, namely D1- and D2-MSNs in the NAc, by stereotaxic injection of Cre-dependent HSV vectors expressing Fosb-targeted ZFPs.

To confirm the Cre-dependent expression of our constructs, we validated the HSV-LS1L-Fosb-ZFP-G9a vector in vivo in WT, D1-Cre, and D2-Cre mice (Figure 1). First, we confirmed viral transduction in both Cre+ and
Cre - neurons by GFP expression, which is driven by a separate, non-Cre-dependent promoter in our viral plasmid (Figures 1a and b). Second, we confirmed Cre-regulated gene expression via coinjection of HSV-LSIL-mCherry, which expresses mCherry selectively in Cre+ neurons, allowing us to identify Cre+ and Cre - neurons. Third, to confirm that only Cre+ (mCherry+) neurons express functional LS1LFosb-ZFP-G9a vectors, we quantified the FosB expression

a
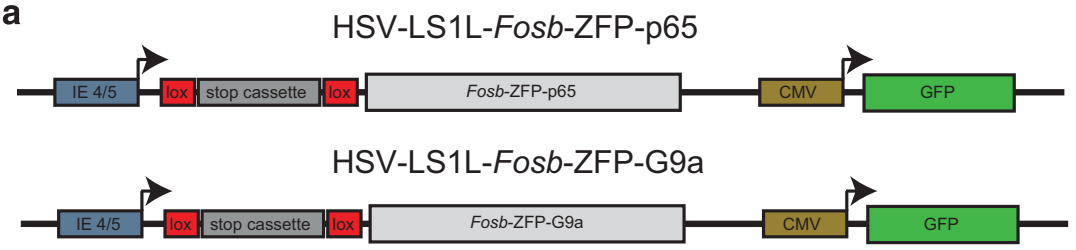

b

GFP
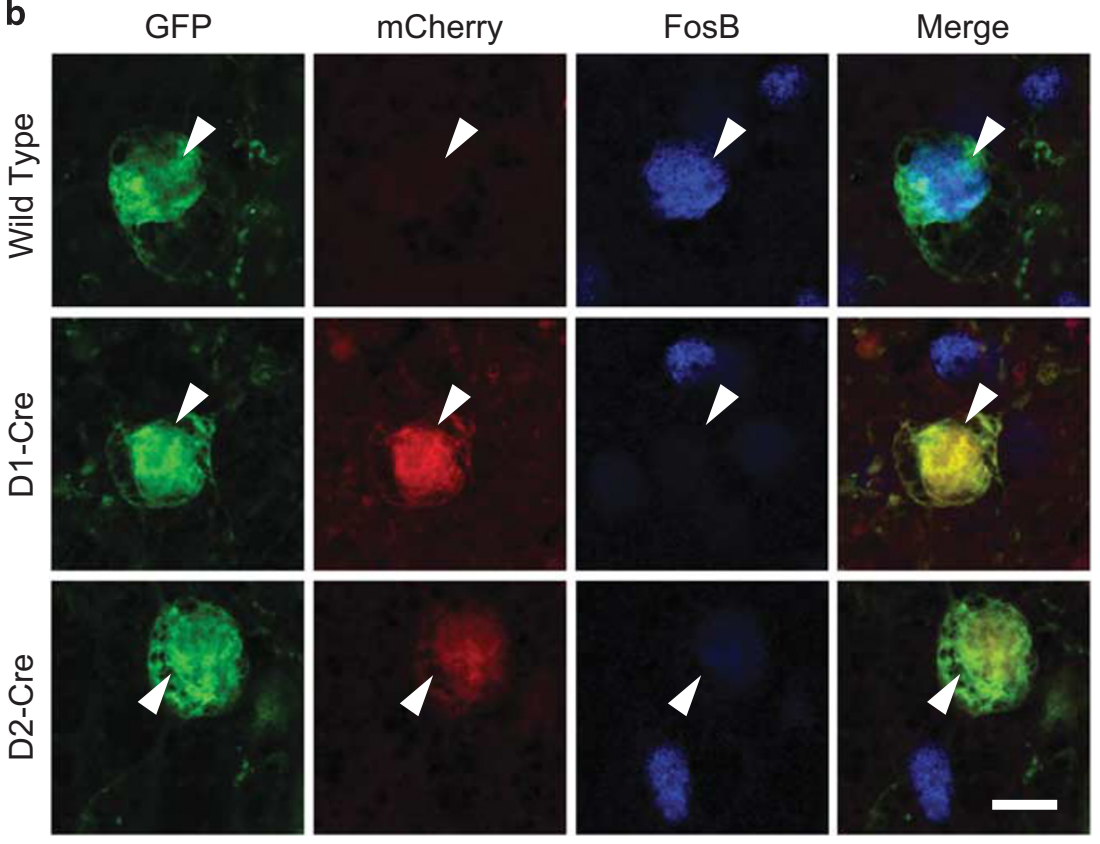

c

d Non-Transduced Cells
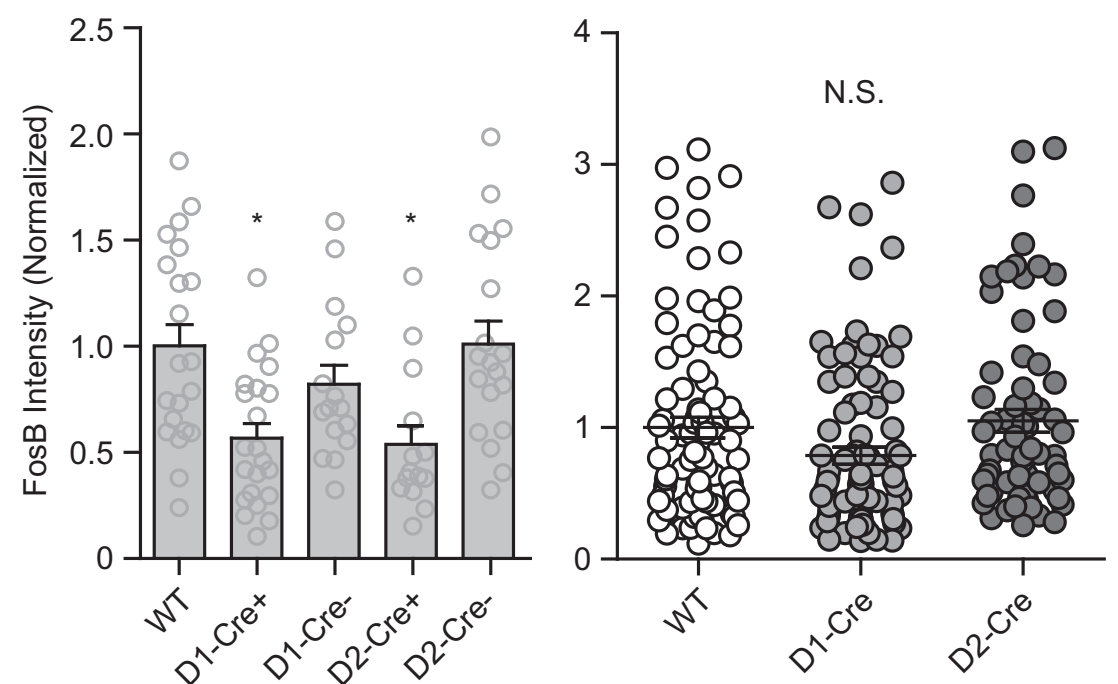
levels in these Cre+ cells. Indeed, we find that only D1- and D2-Cre+ cells, but not Cre - cells, show reduced FosB expression relative to WT levels (Figures $1 \mathrm{~b}$ and $\mathrm{c}$ ). We observe no difference in FosB expression in non-transduced cells in WT, D1-Cre, or D2-Cre mice (Figure 1d and Supplementary Figure 1). These data validate our approach of targeted, cell-type-specific control of the Fosb gene via epigenetic editing through Cre-dependent expression of our engineered ZFP constructs.

\section{Fosb-Targeted Histone Acetylation in D2-MSNs Promotes Susceptibility to Social Stress}

We first investigated the effects of Fosb-targeted histone acetylation exclusively in D2-expressing NAc MSNs. Given that $\Delta$ FosB is induced in D2-MSNs in mice susceptible to CSDS (Lobo et al, 2013), we hypothesized that ZFP-mediated activation in D2-MSNs would be prodepressive. We injected HSV-LS1L-Fosb-ZFP-p65 into the NAc of D2-Cre mice or their WT littermates. Six days after the HSV injection, mice were subjected to one day of subthreshold social defeat stress (Figure $2 \mathrm{~b}$ ), a paradigm that does not produce a depressive, social-avoidant phenotype in control animals (Chaudhury et al, 2013), and would thus be useful to identify a prodepressive effect of D2-MSN-specific Fosb histone acetylation and gene activation. Indeed, we found that WT mice injected with HSV-LS1L-Fosb-ZFP-p65 display a characteristic increase in the amount of time spent in the interaction zone when the novel target mouse is present, indicating the subthreshold social defeat stress was not of sufficient intensity to induce a susceptible, socially avoidant phenotype in control mice. D2-Cre mice injected with HSVLS1L-Fosb-ZFP-p65 do not demonstrate this increased social interaction when a novel target mouse is present (Figure 2c), indicating that D2-MSN-specific histone acetylation at Fosb induces susceptibility to social defeat stress. To more fully explore this possibility, we determined each mouse's social interaction ratio, defined as time spent in the interaction zone when the target mouse is present/absent, and defined the mice as susceptible $($ ratio $<1)$ or resilient $($ ratio $>1$ ) in accordance with previously published methodology (Krishnan et al, 2007). We observed that, while $83 \%$ of WT mice were resilient to subthreshold social defeat stress, only $55 \%$ of D2-Cre littermates were resilient, supporting our hypothesis that histone acetylation exclusively at the Fosb locus within D2-MSNs promotes susceptibility to social defeat stress (Figure 2d).

Mice that exhibit susceptibility to CSDS also display behaviors reminiscent of anxiety and natural reward anhedonia (Berton et al, 2012; Krishnan et al, 2007). To determine whether Fosb gene-specific histone acetylation promoted the full complement of these behavioral abnormalities, we tested animals in the EPM, to study the anxietyrelated effects of this manipulation. We found that Fosbtargeted histone acetylation specifically in D2-MSNs significantly decreased the percent time mice spent exploring the open arms in the EPM (Figure 2e), with no effect on percent time in closed arms, percent time in center, or total locomotor distance traveled during the EPM test (Supplementary Figure 2A). Further, utilizing a sucrose preference paradigm as a measure of anhedonia, we found that Fosb-targeted histone acetylation specifically in D2-
MSNs decreased preference for sucrose consumption relative to WT littermates (Figure 2f). Our manipulations had no effect on the gross body weights of the animals (weights of mice post defeat; WT: $19.0 \pm 0.7 \mathrm{~g}, n=6$; D2-Cre: $17.8 \pm 0.8 \mathrm{~g}, n=11$; expressed as mean \pm SEM; $p>0.05$ by Student's $t$-test). Taken together, these findings indicate that site-specific epigenetic editing of the Fosb gene in a single cell type-increasing permissive histone acetylation in D2-MSNs -is sufficient to promote the full complement of depressiveand anxiety-like behaviors following subthreshold social defeat stress.

\section{Fosb-Targeted Histone Methylation in D2-MSNs Promotes Resilience to Social Stress}

We next investigated the role of Fosb-targeted repressive histone methylation specifically in D2-MSNs in NAc to determine if the results would reflect the converse of that seen following Fosb gene activation in this cell type (Figure 3a). We selected an accelerated defeat paradigm sufficient to produce a depressive-like, social-avoidant phenotype in control animals while matching the time course of HSV expression (Dias et al, 2014). Three days before subjecting mice to accelerated social defeat stress (Figure 3b), we injected the NAc of D2-Cre mice with HSVLS1L-FosB-ZFP-G9a, which methylates Fosb-associated histones (H3K9me2), leading to Fosb gene repression (Heller et al, 2014). We found that WT mice injected with HSVLS1L-FosB-ZFP-G9a and subjected to an accelerated social defeat demonstrated a significant reduction in the amount of time spent in the interaction zone when a novel target mouse is present (Figure 3c). Conversely, D2-Cre mice do not demonstrate a significant reduction in the time spent in the interaction zone, indicating an increased resilience to the social stress (Figure 3c). Analysis of the percentage of susceptible and resilient animals shows an increase in resilience in the population of D2-Cre mice relative to their WT littermates (Figure 3d).

We next sought to determine whether Fosb-specific histone methylation selectively in D2-MSNs was sufficient to repress additional measures of stress susceptibility. We found that there was no difference in open-arm exploratory behavior (Figure 3e), time in closed arms, time in center, or total locomotor distance traveled in the EPM (Supplementary Figure 2B). Note that while both groups of mice display only $5 \%$ exploratory behavior in open arms, which may preclude our ability to measure a further reduction in this measure, previous studies have documented percent time in open-arm below that reported here (Krishnan et al, 2007). We also observed no difference in body weight (weights of mice post defeat; WT: $20.4 \pm 0.7 \mathrm{~g}$, $n=9$; D2-Cre: $18.8 \pm 0.6 \mathrm{~g}, n=9$; expressed as mean \pm SEM; $p>0.05$ by Student's $t$-test). However, consistent with our social interaction observations, mice with targeted histone methylation at the Fosb locus selectively in D2-MSNs displayed a greater preference for sucrose consumption compared with their WT littermates, demonstrating resistance to the anhedonic effects of social defeat (Figure $3 \mathrm{f}$ ). Taken together, these data indicate that Fosb-targeted repressive histone methylation in D2-MSNs confers resilience specifically to the socially avoidant and anhedonic-like elements of social defeat stress. 

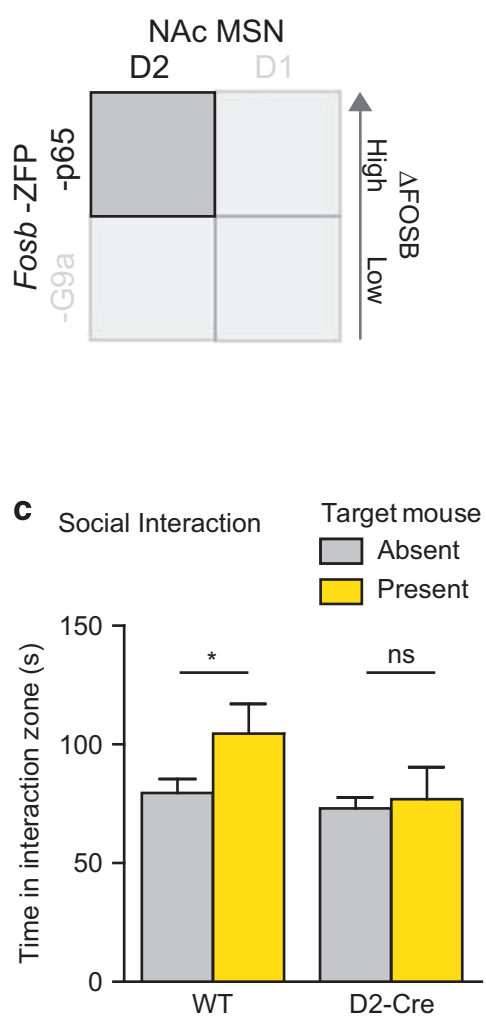

e Elevated Plus Maze

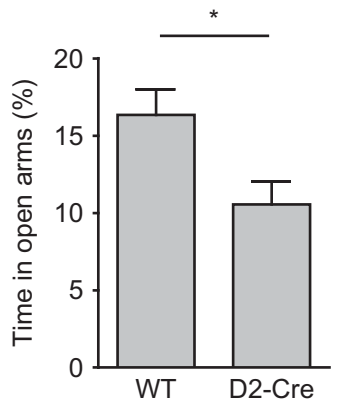

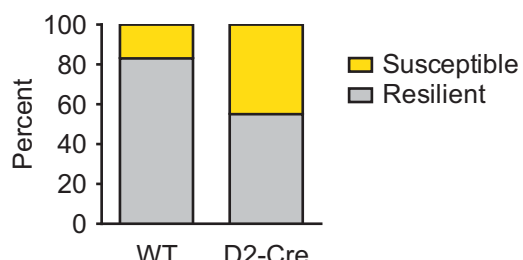

b

SUBTHRESHOLD SOCIAL DEFEAT

reveals increased susceptibility

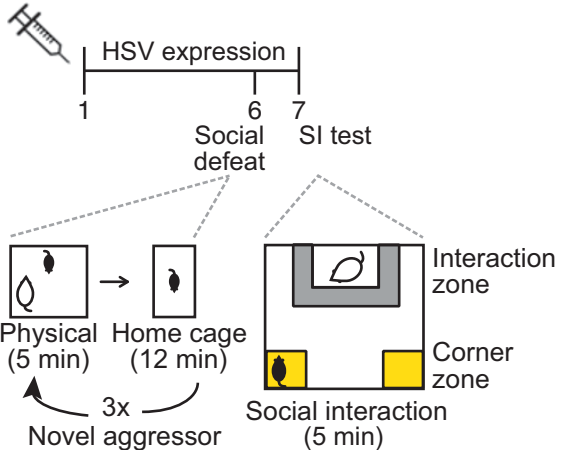

d Susceptibility

WT

f Sucrose Preference

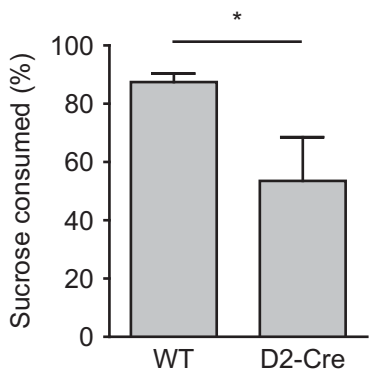

Figure 2 Fosb-ZFP-p65 mediated histone acetylation selectively in D2-medium spiny neurons (MSNs) promotes susceptibility to social stress. (a) The experiments conducted in this study are depicted as a matrix in which the dark grey quadrant specifies the particular experimental mouse and ZFP studied in that figure. The matrix is organized such that the targeted cell type is displayed horizontally and the ZFP construct is displayed vertically. The ZFP effect on $\Delta$ FosB expression is indicated along the right vertical axis. (b) Wild-type (WT) $(n=6)$ or D2-Cre $(n=1 \mathrm{I})$ mice were injected intranucleus accumbens (NAc) with HSV expressing Cre-dependent Fosb-ZFP-p65 and allowed to recover for 6 days before being subjected to a subthreshold social defeat paradigm. After I day of social defeat stress, which consisted of three 5-min bouts with a novel aggressor mouse, mice were tested for stress phenotypes in social interaction, sucrose preference, and elevated plus maze (EPM) tests. (c) Social interaction tests revealed that WT mice subjected to subthreshold social defeat displayed a significantly increased preference for the interaction zone when a novel mouse was present. This increase in social preference was not observed in mice that received Fosb targeted histone acetylation in D2-MSNs, indicating a prosusceptible effect (*⿻ $<0.05$; Student's t-test). (d) Quantification of the social interaction $(\mathrm{SI})$ ratio and determination of the resilience (SI ratio $>$ I) or susceptibility (SI ratio $<1$ ) of each mouse in the WT and D2-Cre populations revealed an increase in susceptibility in the D2-Cre population. (e) D2-Cre mice injected with HSV-LSI L-Fosb-ZFP-p65 spent less time exploring the open arms of the EPM relative to their WT littermates (* $p<0.05$; Student's $t$-test). ( $f$ ) D2-Cre mice injected with HSV-LSI L-Fosb-ZFP-p65 displayed decreased consumption of a $1 \%$ sucrose solution relative to WT mice ( $p<0.05$; Student's $t$-test). Data are presented as mean \pm SEM. NS, not significant.

\section{Fosb-Targeted Histone Acetylation in D1-MSNs Promotes Resilience to Social Stress}

Given that $\triangle$ FosB induction after CSDS occurs specifically in D1-MSNs in resilient mice (Lobo et al, 2013), we hypothesized that ZFP-mediated Fosb activation in D1-MSNs would be antidepressive. We injected HSV-LS1L-FosB-ZFPp65 into the NAc of D1-Cre mice or their WT littermates 3 days before subjecting mice to 4 days of accelerated social defeat stress (Figures $4 \mathrm{a}$ and $\mathrm{b}$ ). As before, this paradigm produced a social avoidance phenotype in WT 
a
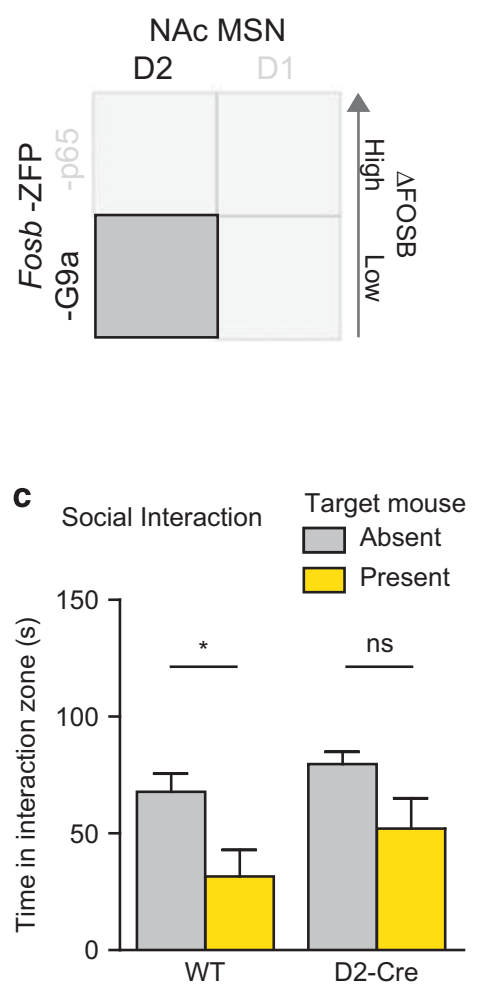

e Elevated Plus Maze

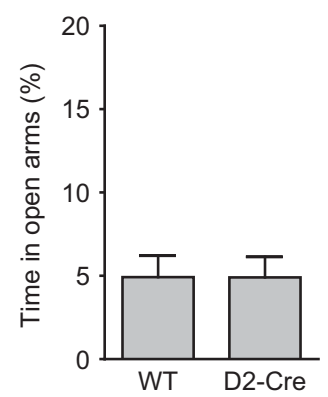

b

ACCELERATED SOCIAL DEFEAT

reveals decreased susceptibility

Y

HSV expression

Social defeat SI test

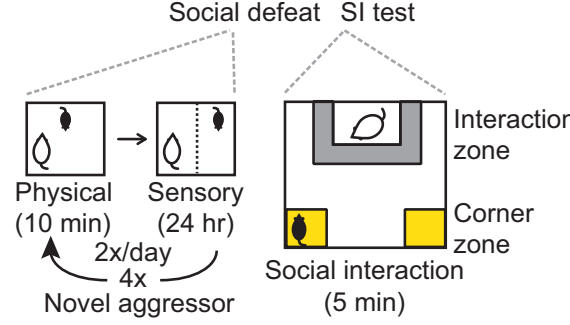

d

Susceptibility

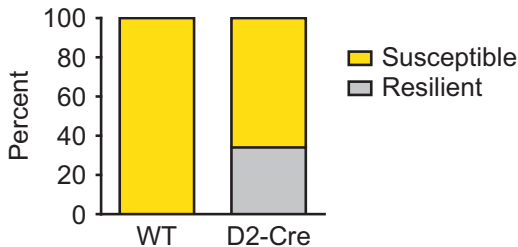

f

Sucrose Preference

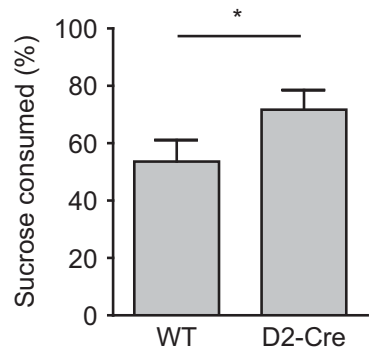

Figure 3 Fosb-ZFP-G9a mediated histone methylation selectively in D2-medium spiny neurons (MSNs) promotes resilience to social stress. (a) See description in Figure 2a. (b) Wild-type (WT) $(n=9)$ or D2-Cre $(n=9)$ mice were injected intranucleus accumbens (NAc) with herpes simplex virus (HSV) expressing Cre-dependent Fosb-ZFP-G9a and allowed to recover for $48 \mathrm{~h}$ before being subjected to an accelerated social defeat paradigm. Following 4 days of social defeat stress, mice were tested for stress phenotypes in social interaction, sucrose preference and elevated plus maze (EPM) tests. (c) Social interaction tests revealed that stressed WT mice spent significantly less time in the interaction zone when a novel mouse was present, whereas mice that received Fosb targeted histone methylation in D2-MSNs did not, indicating a proresilience effect (* $p<0.05$; Student's t-test). (d) Quantification of the social interaction (SI) ratio revealed a greater percentage of resilient animals in the D2-Cre population. (e) WT and D2-Cre mice displayed no difference in anxiety-like behavior as determined by percent time exploring the open arm of the EPM. (f) D2-Cre mice injected with HSV-LSI L-Fosb-ZFP-G9a demonstrated a greater preference for consumption of a $1 \%$ sucrose solution (* $p<0.05$; Student's $t$-test). Data are presented as mean \pm SEM. NS, not significant.

animals (Figure 4c). Importantly, targeted histone acetylation at the Fosb gene in D1-MSNs promoted resilience to social defeat stress, as seen by a decrease in social avoidance behavior in the D1-Cre mice (Figure 4c) and a shift towards greater resilience in the D1-Cre mice as quantified by the percentage of animals displaying a resilient or susceptible phenotype in WT and D1-Cre populations (Figure 4d).

To determine whether Fosb-associated histone acetylation in D1-MSNs also protected animals from the anxiety-like effects of social defeat stress, we tested mice in the EPM. Surprisingly, we found a significant decrease in the amount of time D1-Cre mice spent exploring the open arms (Figure 4e) with no effect on percent time in closed arms, percent time in center, or total locomotor distance traveled during the EPM test (Supplementary Figure 2C). Using the sucrose preference paradigm as a measure of anhedonia, we observed that Fosb-targeted histone acetylation in D1-MSNs led to a significant increase in preference for sucrose relative to plain water (Figure $4 \mathrm{f}$ ) 


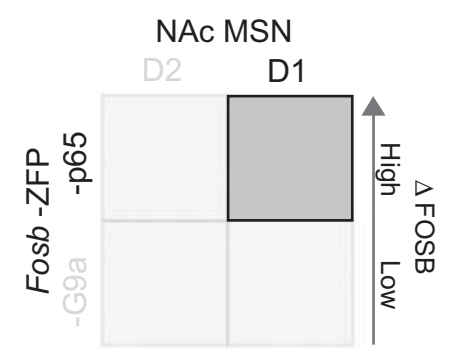

reveals decreased susceptibility
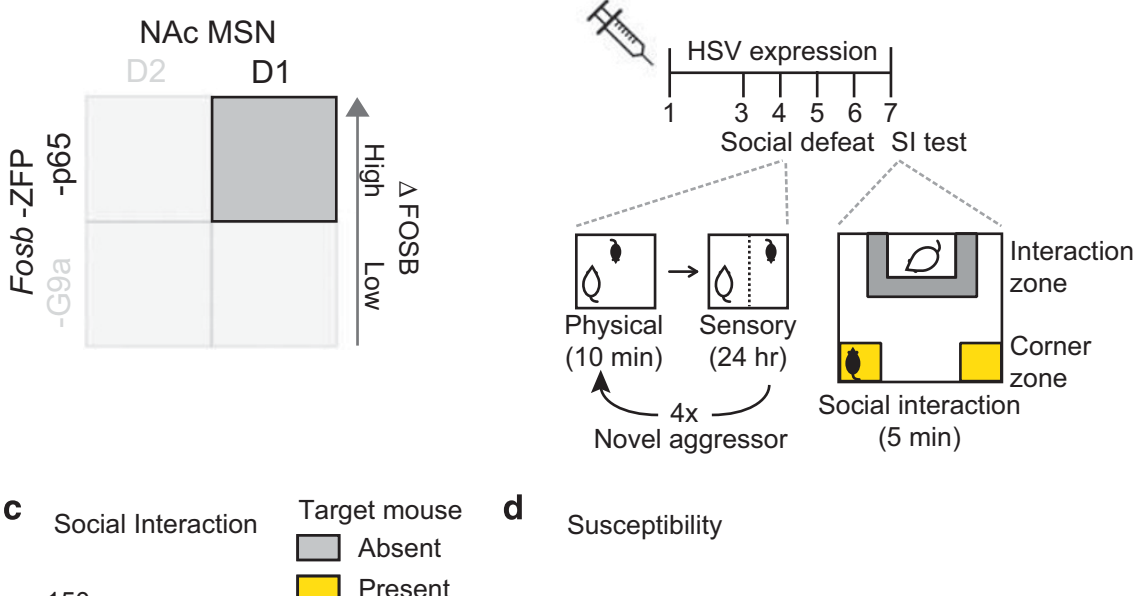

d Susceptibility
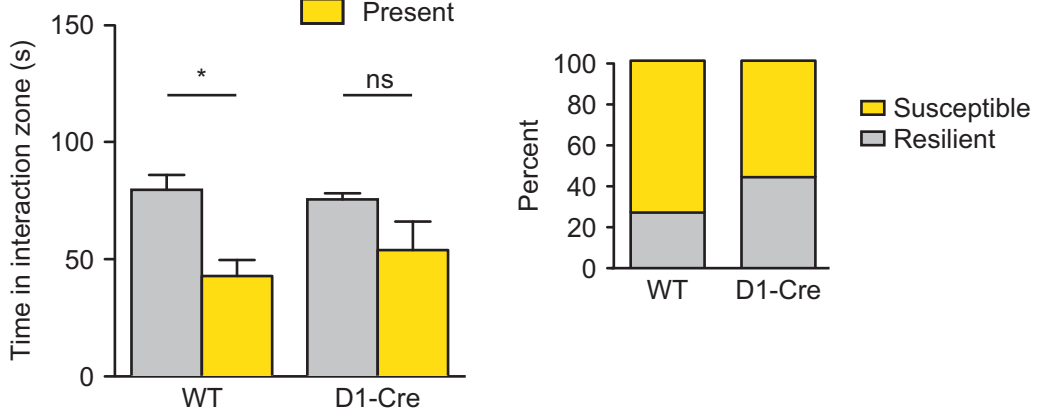

e Elevated Plus Maze
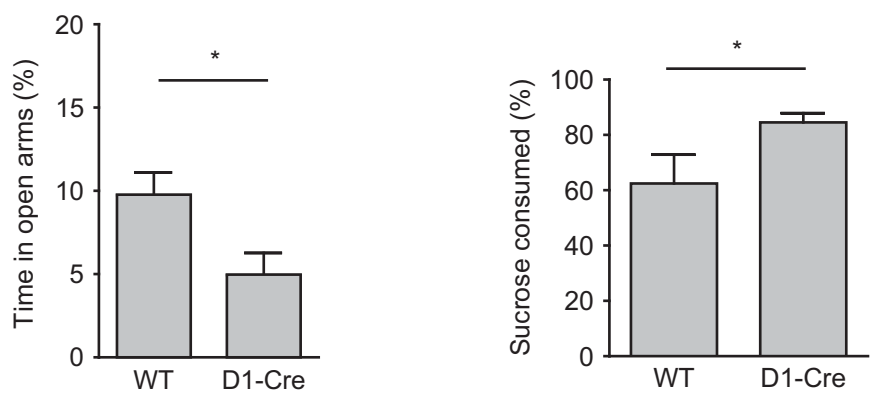

Figure 4 Fosb-ZFP-p65 mediated histone acetylation selectively in DI-medium spiny neurons (MSNs) promotes resilience to social stress. (a) See description in Figure 2a. (b) Wild-type (WT) $(n=1 \mathrm{I})$ or DI-Cre $(n=9)$ mice were injected intranucleus accumbens (NAc) with herpes simplex virus (HSV) expressing Cre-dependent Fosb-ZFP-p65 and allowed to recover for $48 \mathrm{~h}$ before being subjected to an accelerated social defeat paradigm. Following 4 days of social defeat stress, mice were tested for stress phenotypes in social interaction, sucrose preference, and elevated plus maze (EPM) tests. (c) Social interaction tests revealed that stressed WT mice spent significantly less time in the interaction zone when a novel mouse was present, whereas mice that received Fosb targeted histone acetylation in DI-MSNs did not, indicating a proresilience effect (* $p<0.05$; Student's t-test). (d) Quantification of the social interaction (SI) ratio revealed a greater percentage of resilient animals in the DI-Cre population. (e) Relative to WT mice, DI-Cre mice injected with HSV-LSI L-Fosb-ZFPp65 spent less time exploring the open arm of the EPM (*p <0.05; Student's t-test). (f) DI-Cre mice injected with HSV-LSI L-Fosb-ZFP-p65 demonstrated a greater preference for consumption of a $1 \%$ sucrose solution $(* p<0.05$; Student's $t$-test). Data are presented as mean \pm SEM. NS, not significant.

without affecting body weight (weights of mice post defeat; WT: $\quad 22.5 \pm 0.4 \mathrm{~g}, \quad n=11 ; \quad$ D1-Cre: $21.6 \pm 0.3 \mathrm{~g}, \quad n=9$; expressed as mean \pm SEM; $p>0.05$ by Student's $t$-test). Taken together, these findings indicate that site-specific permissive histone acetylation of the Fosb gene in D1-MSNs protects against the social avoidance and anhedonic phenotypes induced by social defeat, but not anxiety traits as measured by exploratory behavior in the EPM (see Discussion).

\section{Fosb-Targeted Histone Methylation in D1-MSNs Promotes Susceptibility to Social Stress}

To complete our systematic interrogation of targeted histone acetylation or methylation at the Fosb gene in each NAc MSN cell type, we tested whether repressive histone methylation at Fosb within D1-MSNs exacerbates the consequences of social defeat stress. We injected the NAc of D1-Cre or WT mice with HSV-LS1L-FosB-ZFP-G9a 6 days before subjecting them to subthreshold social defeat 
a

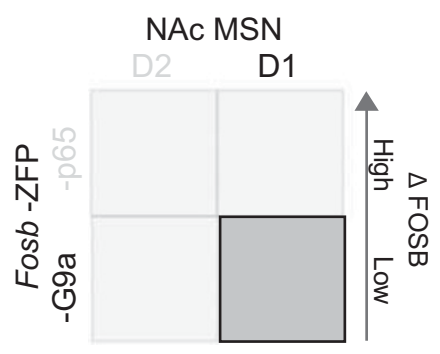

b SUBTHRESHOLD SOCIAL DEFEAT reveals increased susceptibility

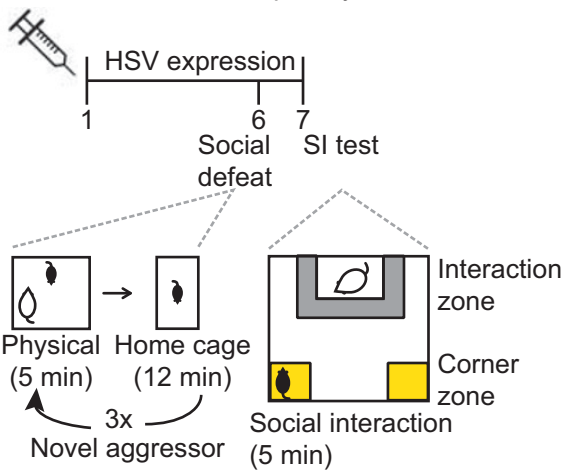

Susceptibility
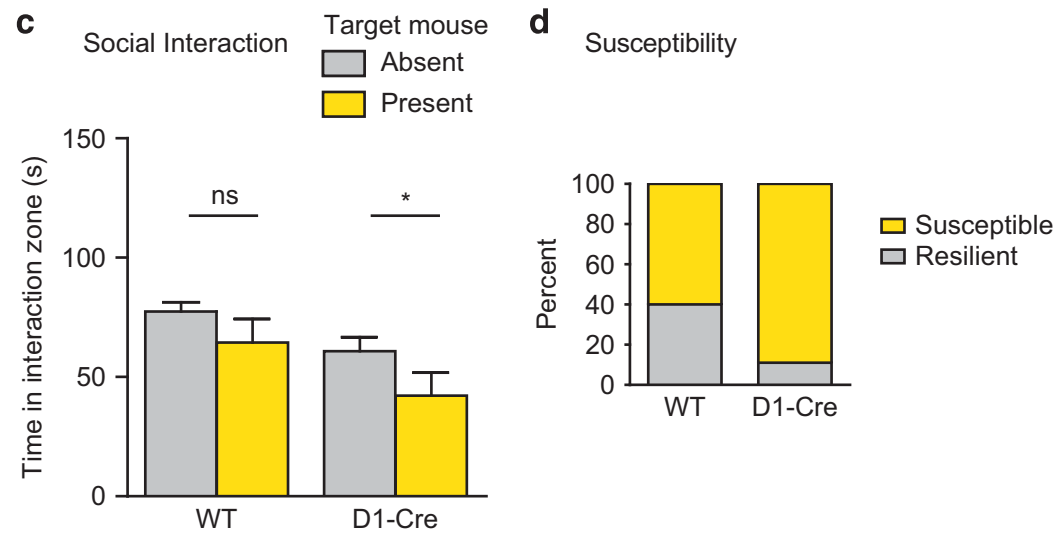

e Elevated Plus Maze

f Sucrose Preference
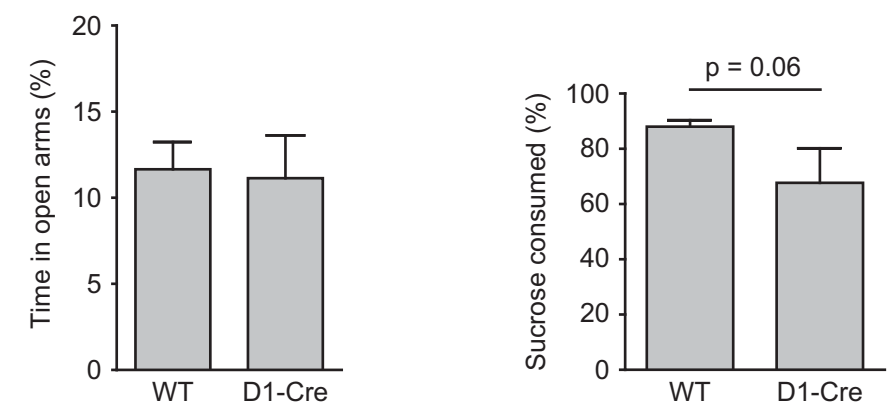

Figure 5 Fosb-ZFP-G9a mediated histone methylation selectively in DI-medium spiny neurons (MSNs) promotes susceptibility to social stress. (a) See description in Figure 2a. (b) Wild-type (WT) $(n=10)$ or D I-Cre $(n=10)$ mice were injected intranucleus accumbens (NAc) with herpes simplex virus (HSV) expressing Cre-dependent Fosb-ZFP-G9a and allowed to recover for 6 days before being subjected to a subthreshold social defeat paradigm. After one day of social defeat stress, mice were tested for stress phenotypes in social interaction, sucrose preference, and elevated plus maze (EPM) tests. (c) Social interaction tests revealed that mice that received Fosb-targeted histone methylation in D I-MSNs subjected to subthreshold social defeat spent significantly less time in the interaction zone when a novel mouse was present (a prosusceptible effect), whereas their WT littermates did not (* $p<0.05$; Student's $t$-test). (d) Quantification of the social interaction (SI) ratio revealed a greater percentage of susceptible animals in the DI-Cre population than in the WT littermate population. (e) WT and DI-Cre mice injected with HSV-LSIL-Fosb-ZFP-G9a exhibited no significant difference in anxiety-like symptoms as assessed by percent time spent on the open arms of the EPM. (f) DI-Cre mice injected with HSV-LSI L-Fosb-ZFP-p65 demonstrated a trend towards a lower preference for consumption of a $1 \%$ sucrose solution $(p=0.06$; Student's $t$-test). Data are presented as mean \pm SEM. NS, not significant.

stress (Figures $5 \mathrm{a}$ and $\mathrm{b}$ ). In these mice, we observed that D1-MSN-specific histone methylation at Fosb led to a significant decrease in the amount of time spent in the interaction zone during the interaction test, whereas WT littermates showed no reduction in their social interaction scores (Figure 5c). Relative to WT mice, we did observe a reduction in the amount of time that D1-Cre mice spent in the interaction zone when the target mouse was absent
( $p<0.05$ by Student's $t$-test). This effect is not due to an alteration in basal locomotor behavior in D1-Cre mice (total locomotor distance moved when target mouse was absent: WT: $1382 \pm 54 \mathrm{~cm}, n=10$; D1-Cre: $1507 \pm 70 \mathrm{~cm}, n=10$; expressed as mean \pm SEM; $p>0.05$ by Student's $t$-test). Importantly, upon stress exposure, WT and D1-Cre mice differentially manifest stress responses as seen in time spent in the interaction zone when the target mouse is present. 
This difference in stress response was revealed further when we observed that the percentage of mice displaying resilience to social defeat stress decreases in the D1-Cre population (Figure 5d).

We observed no difference between WT and D1-Cre mice in anxiety-like behaviors, as quantified as percentage of time in open arm of EPM (Figure 5e), or time in closed arm, center, or total locomotion scores (Supplementary Figure 2D). Further, we saw no changes in gross body weight (weights of mice post defeat; WT: $19.9 \pm 0.4 \mathrm{~g}, n=10$; D1-Cre: $20.2 \pm 0.6 \mathrm{~g}, \quad n=10$; expressed as mean $\pm \mathrm{SEM}$; $p>0.05$ by Student's $t$-test). However, when we investigated the effect of D1-MSN-specific histone methylation at the Fosb gene in the development of anhedonic-like phenotypes, we observed that mice expressing ZFP-Fosb-G9a in D1-MSNs demonstrated a trend towards a decreased preference for consuming a sucrose solution (Figure 5f). Taken together, we found that increased repressive histone methylation at the Fosb gene solely in D1-MSNs is sufficient to exacerbate the deficits in social interaction induced by social defeat stress.

\section{DISCUSSION}

This is the first study to usee cell-type-specific targeted epigenetic editing in vivo in a neuropsychiatric syndrome model. While it is well established that epigenetic modifications contribute to depression (Berton et al, 2012; Pena et al, 2014; Sun et al, 2013), it has been difficult to define the exact cell types in which these modifications function. Using our innovative approach, we analyzed the cellular specificity of Fos $b$ transcription in controlling susceptibility $v s$ resilience to social defeat stress. These findings are particularly relevant to our understanding of the causal role of epigenetic remodeling in stress responses, which is critical for the development of novel therapeutics for stress-related neuropsychiatric disorders.

We targeted Fosb epigenetic remodeling to distinct subpopulations of NAc neurons, given emerging data on the distinct roles of the D1 and D2 subtypes of MSNs. In particular, a prior study from our group found differences in the extent of $\Delta$ FosB induction in the two cell types in response to several forms of chronic pharmacological (cocaine, haloperidol, $\Delta$ 9-THC, heroin, morphine), emotional (environmental enrichment, calorie restriction, sucrose two-bottle choice, social defeat, fluoxetine), and optogenetic stimuli (Lobo et al, 2013). Examination of the effect of optogenetic activation of various NAc afferent regions in these two mouse lines found different levels of $\Delta$ FosB induction in the two cell types as a function of input stimulation (Lobo et al, 2013). Interestingly, an additional study found that optogenetic stimulation of distinct populations of dynorphinergic cells also show specific regulation of emotional behavior. That is, stimulation of dynorphinergic cells in the ventral NAc elicited robust conditioned and real-time aversive behavior, while photostimulation of dorsal NAc dynorphin cells was positively reinforcing (Al-Hasani et $a l, 2015)$. Taken together, these studies indicate that cell-type-specific regulation of emotional behavior extends beyond MSNs to dynorphinergic, and likely other, cell types. To complement these optogenetic studies, we selected to epigenetically regulate Fosb expression in each of the two MSN cell types and examine depressive-like behavior.

Prior studies have described an increase in $\Delta$ FosB levels in the NAc of mice both susceptible and resilient to CSDS, with a larger increase seen in the resilient population (Vialou et al, $2010 \mathrm{~b})$. In addition, while inducible overexpression of $\Delta \mathrm{FosB}$ in all NAc neurons was shown to mediate directly a state of resilience in the social interaction test (Vialou et al, 2010b), subsequent studies indicated that the observed increase in $\Delta$ FosB levels in the NAc of resilient mice occurs solely in D1-MSNs, whereas D2-MSNs display increased $\Delta$ FosB levels specifically in susceptible mice (Lobo et al, 2013). Our study used targeted manipulation of the chromatin state at the Fosb gene to regulate $\triangle F O s B$ expression in each cell type for a direct examination of the causal role of epigenetic modulation in each MSN type in mediating a susceptible $v s$ resilient phenotype

Social defeat stress produces several depressive-like behaviors in mice, including social avoidance and anhedonia, as well as anxiety-like symptoms (Berton et al, 2006; Donahue et al, 2014; Krishnan et al, 2007). We found that activation of Fosb expression via histone acetylation in D2-MSNs is causal in potentiating a full complement of depressive- and anxiety-like behaviors induced by social stress, including social avoidance in the social interaction test, and increased anhedonia- (sucrose preference) and anxiety- (EPM) like responses. Conversely, Fosb-targeted repressive histone methylation in D2-MSNs promotes resilience to social defeat stress, as reflected by protection against the social avoidance and anhedonic phenotypes associated with this paradigm. Interestingly, there was no effect on the exploratory behavior of these animals, suggesting a functional separation between depressive- and anxiogenic-like traits associated with social defeat stress. Such separation of phenotype has been observed previously (Krishnan et al, 2007), in that resilient mice do not display depressive-like behavior, as measured by social interaction and sucrose preference, but do display increased anxiety-like behaviors, measured as decreased exploration of the open arms in the EPM (Krishnan et al, 2007). This observed separation of behavioral phenotype may account for the inconsistent expression of anxiety-like behavior in the EPM following cell-type-specific epigenetic editing of Fosb. Specifically, while two distinct Fosb-targeted histone modifications were associated with increased social avoidance behavior, only acetylation of Fosb in the D2-MSNs, but not methylation of Fosb in D1-MSNs, was associated with decreased open-arm exploration in EPM. Along similar lines, activation of Fosb expression in the D1-MSNs promoted resilience in the social interaction test and protected against anhedonia, as predicted by its expression pattern following social defeat stress (Lobo et al, 2013), but paradoxically caused an increase in anxiety-like responses. Repression of Fosb expression via histone methylation in D1-MSNs promoted susceptibility to social avoidance, but had less of an effect on EPM and sucrose preference behavior. While these results illuminate the differential contributions of cell-type-specific HPTMs at the Fosb locus in driving depressive-like behaviors, the inconsistent results in anxiety-like behaviors may be due to similar, yet undefined, mechanisms regulating the emergence of resilient and susceptible phenotypes. Finally, it is important to note 
that the behavioral measurements were taken at different times following viral injection, that is, EPM was tested 7-8 days following HSV delivery. It is therefore possible that the inconsistent behavior expressed at this time point is due to diminishing HSV expression. This is not likely to be the case, however, given that HSV-ZFP expression consistently regulates behaviors measured 7-10 days after viral injection (Heller et al, 2014, 2016), indicating that the epigenetic manipulations are stable beyond the maximal expression of HSV. Indeed, we do observe decreased open-arm exploratory behavior in mice expressing FosB-ZFP-p65 in D2-MSNs, revealing a novel functional role for epigenetic modulation of Fosb within D2-MSNs in driving both depressive- and anxiety-like behaviors induced by social stress.

It is important to note that the behavioral effect sizes observed in this study are small relative to more traditional overexpression and knockout strategies. This difference can be attributed to the subtlety of our manipulation, that is, the targeting of a single epigenetic modification to a single locus in a single-cell type. This mode of gene regulation drives physiologically relevant changes in expression level $(\sim 1.2-$ 2.0-fold) (Gilbert et al, 2013; Heller et al, 2014, 2016) that are lower than that seen with exogenous gene expression or gene knockout (Chen et al, 1998). Transcriptional activation via HSV expression of Fosb-ZFP-p65 results in a $\sim 2$-fold induction of $\Delta$ Fosb mRNA in the NAc (Heller et al, 2014), which is comparable to the twofold induction in FosB +-immunoreactive cells following social defeat stress (Lobo et al, 2013; Vialou et al, 2010b). This is markedly different from the expression levels seen in transgenic mice or virally treated mice with inducible $\Delta$ FosB overexpression, in which the degree of $\Delta$ FosB induction far exceeds levels of the endogenous protein induced in brain by numerous treatments (Chen et al, 1998). Thus, our tools make it possible to mirror the endogenous physiology of stress responses by emulating natural epigenetic mechanisms of inducing physiologically relevant transcriptional events within specific cell populations and avoiding potential artifacts seen with non-physiological degrees of gene manipulation.

In addition to more closely modeling physiological levels of gene expression, our approach is the first to elucidate the causal relevance of naturally occurring, stress-induced modes of epigenetic regulation at a single gene. The importance of parsing cell- and gene-specific functions of HPTMs is underscored by the fact that epigenetic alterations associated with chronic stimuli also appear to be cell-type-specific. An immunohistochemical examination of global histone modifications in the striatum following chronic cocaine treatment found that acetylation of histone $\mathrm{H} 4$ lysine 5 is increased specifically in D1-MSNs, while $\mathrm{H} 3 \mathrm{~K} 14 \mathrm{Ac}$ and $\mathrm{H} 4 \mathrm{~K} 8 \mathrm{Ac}$ are enriched in D2-MSNs (Jordi et al, 2013). Histone methylation appeared to be upregulated exclusively in D1-MSNs (Jordi et al, 2013). Interestingly, D1- or D2-MSN-targeted deletion of G9a, which, as noted earlier, catalyzes H3K9me2, alters behavioral responsiveness to several pharmacological stimuli in a cell-type-specific manner (Schaefer et al, 2009). Additionally, while repeated cocaine exposure reduces G9a expression in both D1- and D2-MSNs, conditional G9aknockout or adult G9a overexpression in D1- vs D2-MSNs produced opposing behavioral responses to cocaine treatment (Maze et al, 2014). One prior study investigating the connection between cocaine-repressed histone methylation in MSNs and subsequent stress exposure found that global reduction in $\mathrm{G} 9 \mathrm{a}$ and $\mathrm{H} 3 \mathrm{~K} 9 \mathrm{me} 2$ levels following chronic cocaine exposure increased susceptibility to social defeat stress (Covington et al, 2011). Paradoxically, we have measured enrichment of $\mathrm{H} 3 \mathrm{~K} 9 \mathrm{me} 2$ at the Fosb locus in depressed human NAc and found that Fosb-targeted $\mathrm{H} 3 \mathrm{~K} 9 \mathrm{me} 2$ in NAc increases susceptibility to social defeat stress in mice (Heller et al, 2014). To determine whether these differences in stress regulation by $\mathrm{H} 3 \mathrm{~K} 9 \mathrm{me} 2$ are cell-type-specific, in this study, we found that increased H3K9me2 at the Fosb gene specifically in D1-MSNs promotes susceptibility to social defeat stress, whereas Fosb-targeted $\mathrm{H} 3 \mathrm{~K} 9 \mathrm{me} 2$ in $\mathrm{D} 2-\mathrm{MSN}$ increases resilience to social defeat stress. Taken together, these results demonstrate differences in the behavioral effects of global epigenetic modifications and targeted epigenetic editing at single loci within specific cell types. A cell-type-specific examination of Fosb-specific histone modifications would greatly enhance our understanding of the relevance of global $v s$ gene-specific histone modifications within specific cell populations. Technical innovations that enable ChIP following fluorescence-activated cell sorting (FACS) (Finegersh and Homanics, 2016; Mitchell et al, 2017; von Schimmelmann et al, 2016) as well as single-cell ChIP sequencing (Rotem et al, 2015) are necessary to elucidate the cell-type-specific mechanisms of Fosb gene expression in regulating stress- and depressive-like behavior.

Since this study was designed to test the hypothesis that cell-type-specific epigenetic regulation of Fosb differentially mediates responses to stress exposure, we did not examine the role, if any, of FosB $/ \Delta$ FosB on basal emotional behavior. Further study using this paradigm may uncover a role for cell-type-specific epigenetic regulation of Fosb in the emotional behavior of stress-naïve mice. Regarding the utility of this approach for studying baseline behaviors, it is important to note that investigators have observed some differences in baseline behavior between the two Cretransgenic lines used in this study, despite the fact that they share the same genetic background (Kramer et al, 2011). For example, we found that the baseline open-arm exploratory behavior (Figures $3 \mathrm{e}$ and $4 \mathrm{e}$ ), baseline social interaction time (Figures $2 \mathrm{c}$ and $5 \mathrm{c}$ ), and percent resilient (Figures $2 \mathrm{~d}$ and $5 \mathrm{~d}$ ) differ between WT littermates of D1- and D2-Cre transgenic animals. To avoid any confounds due to strain differences, all comparisons in this study are thus made within strain between hemizogous transgenic and wild-type littermates.

Given the role of $\Delta$ FosB as a transcription factor, it is likely that the distinct phenotypes revealed by our manipulations are due to unique subsets of downstream gene targets for $\Delta$ FosB in D1- vs D2-MSNs. Indeed, it is the stable accumulation of $\Delta$ FosB that distinguishes its transcriptional function from more labile members of the Fos family of immediate early genes (Nestler, 2008). For example, $\Delta$ FosB accumulation is known to regulate downstream target genes associated with synaptic plasticity, such as the AMPA glutamate receptor subunit GluA2 (Vialou et al, 2010b) and calcium/calmodulin-dependent protein kinase II (Robison et al, 2013, 2014). Moreover, $\Delta$ FosB overexpression reduces AMPA current in D1-MSNs, but not D2-MSNs, as would be expected from GluA2 induction (Grueter et al, 2013). Further investigation of the expression of glutamate and GABA receptors, as well as transcription factors 
regulating their expression, will be needed to elucidate the difference in $\Delta$ FosB-target gene regulation following specific modes of epigenetic regulation of the Fosb locus in distinct cell types. Furthermore, western blot analysis may reveal celltype-specific differences in the post-translational modifications of $\triangle$ FosB known to modulate its function as a transcription factor (Cates et al, 2014). In addition to downstream molecular targets, the regulation of Fosb in each of the MSN projection neurons may have distinct physiological effects on basal dopaminergic tone of VTA neurons. This difference in dopaminergic tone would then be amplified through the opposite response in the two MSNs to dopamine signaling, that is, enhanced glutamatergic activation of D1-MSNs and reduced glutamatergic activation of D2-MSNs (Gerfen and Surmeier, 2011; Surmeier et al, 2007). Thus, in addition to the Fosb-mediated MSN inhibitory tone, regulation of dopaminergic signaling may contribute to the behavioral phenotypes observed.

Locus-specific epigenetic remodeling allows us to model closely the subtle changes in gene expression that underlie mood regulation. Cell-type-specific epigenetic remodeling of a single gene locus allows direct examination of the causal role of such changes in depression-related behavioral abnormalities. We found that, in the context of Fosb-targeted HPTMs in MSN subtypes, these subtle manipulations reveal opposing influences on stress susceptibility depending on the cell type in which they occur. This observation serves to increase the resolution of our understanding of the causal epigenetic mechanisms that contribute to depression-related behaviors.

\section{FUNDING AND DISCLOSURE}

This work was supported by grants from NIDA (R37DA008227) and NIMH (P50MH096890) to EJN and The Robin Chemers Neustein Postdoctoral Fellowship at Mount Sinai to EAH. The authors declare no conflict of interest.

\section{REFERENCES}

Al-Hasani R, McCall JG, Shin G, Gomez AM, Schmitz GP, Bernardi JM et al (2015). Distinct subpopulations of nucleus accumbens dynorphin neurons drive aversion and reward. Neuron 87: 1063-1077.

Barrot M, Olivier JD, Perrotti LI, DiLeone RJ, Berton O, Eisch AJ et al (2002). CREB activity in the nucleus accumbens shell controls gating of behavioral responses to emotional stimuli. Proc Natl Acad Sci USA 99: 11435-11440.

Bateup HS, Svenningsson P, Kuroiwa M, Gong S, Nishi A, Heintz N et al (2008). Cell type-specific regulation of DARPP-32 phosphorylation by psychostimulant and antipsychotic drugs. Nat Neurosci 11: 932-939.

Berton O, Hahn CG, Thase ME (2012). Are we getting closer to valid translational models for major depression? Science 338: 75-79.

Berton O, McClung CA, Dileone RJ, Krishnan V, Renthal W, Russo SJ et al (2006). Essential role of BDNF in the mesolimbic dopamine pathway in social defeat stress. Science 311: 864-868.

Cates HM, Thibault M, Pfau M, Heller E, Eagle A, Gajewski P et al (2014). Threonine 149 phosphorylation enhances DeltaFosB transcriptional activity to control psychomotor responses to cocaine. J Neurosci 34: 11461-11469.
Chaudhury D, Walsh JJ, Friedman AK, Juarez B, Ku SM, Koo JW et al (2013). Rapid regulation of depression-related behaviours by control of midbrain dopamine neurons. Nature 493: 532-536.

Chen J, Kelz MB, Zeng G, Sakai N, Steffen C, Shockett PE et al (1998). Transgenic animals with inducible, targeted gene expression in brain. Mol Pharmacol 54: 495-503.

Colby CR, Whisler K, Steffen C, Nestler EJ, Self DW (2003). Striatal cell type-specific overexpression of DeltaFosB enhances incentive for cocaine. J Neurosci 23: 2488-2493.

Covington HE 3rd, Maze I, Sun H, Bomze HM, DeMaio KD, $\mathrm{Wu}$ EY et al (2011). A role for repressive histone methylation in cocaine-induced vulnerability to stress. Neuron 71: 656-670.

Dias C, Feng J, Sun H, Shao NY, Mazei-Robison MS, Damez-Werno $\mathrm{D}$ et al (2014). beta-catenin mediates stress resilience through Dicer1/microRNA regulation. Nature 516: 51-55.

Donahue RJ, Muschamp JW, Russo SJ, Nestler EJ, Carlezon WA Jr (2014). Effects of striatal DeltaFosB overexpression and ketamine on social defeat stress-induced anhedonia in mice. Biol Psychiatry 76: $550-558$.

Finegersh A, Homanics GE (2016). Chromatin immunoprecipitation and gene expression analysis of neuronal subtypes after fluorescence activated cell sorting. J Neurosci Methods 263: 81-88.

Gerfen CR, Surmeier DJ (2011). Modulation of striatal projection systems by dopamine. Annu Rev Neurosci 34: 441-466.

Gilbert LA, Larson MH, Morsut L, Liu Z, Brar GA, Torres SE et al (2013). CRISPR-mediated modular RNA-guided regulation of transcription in eukaryotes. Cell 154: 442-451.

Graybiel AM (2000). The basal ganglia. Curr Biol 10: R509-R511.

Grueter BA, Robison AJ, Neve RL, Nestler EJ, Malenka RC (2013). FosB differentially modulates nucleus accumbens direct and indirect pathway function. Proc Natl Acad Sci USA 110: 1923-1928.

Hasbi A, Fan T, Alijaniaram M, Nguyen T, Perreault ML, O'Dowd BF et al (2009). Calcium signaling cascade links dopamine D1-D2 receptor heteromer to striatal BDNF production and neuronal growth. Proc Natl Acad Sci USA 106: 21377-21382.

Heller EA, Cates HM, Pena CJ, Sun H, Shao N, Feng J et al (2014). Locus-specific epigenetic remodeling controls addiction- and depression-related behaviors. Nat Neurosci 17: 1720-1727.

Heller EA, Hamilton PJ, Burek DD, Lombroso SI, Pena CJ, Neve RL et al (2016). Targeted epigenetic remodeling of the Cdk5 gene in nucleus accumbens regulates cocaine- and stress-evoked behavior. J Neurosci 36: 4690-4697.

Hope BT, Nye HE, Kelz MB, Self DW, Iadarola MJ, Nakabeppu Y et al (1994). Induction of a long-lasting AP-1 complex composed of altered Fos-like proteins in brain by chronic cocaine and other chronic treatments. Neuron 13: 1235-1244.

Jordi E, Heiman M, Marion-Poll L, Guermonprez P, Cheng SK, Nairn AC et al (2013). Differential effects of cocaine on histone posttranslational modifications in identified populations of striatal neurons. Proc Natl Acad Sci USA 110: 9511-9516.

Kelz MB, Chen J, Carlezon WA Jr, Whisler K, Gilden L, Beckmann AM et al (1999). Expression of the transcription factor deltaFosB in the brain controls sensitivity to cocaine. Nature 401: 272-276.

Kramer PF, Christensen CH, Hazelwood LA, Dobi A, Bock R, Sibley DR et al (2011). Dopamine D2 receptor overexpression alters behavior and physiology in Drd2-EGFP mice. J Neurosci 31: 126-132.

Krishnan V, Han MH, Graham DL, Berton O, Renthal W, Russo SJ et al (2007). Molecular adaptations underlying susceptibility and resistance to social defeat in brain reward regions. Cell 131: 391-404.

Kupchik YM, Brown RM, Heinsbroek JA, Lobo MK, Schwartz DJ, Kalivas PW (2015). Coding the direct/indirect pathways by D1 and D2 receptors is not valid for accumbens projections. Nat Neurosci 18: 1230-1232.

Liu PQ, Rebar EJ, Zhang L, Liu Q, Jamieson AC, Liang Y et al (2001). Regulation of an endogenous locus using a panel of 
designed zinc finger proteins targeted to accessible chromatin regions. Activation of vascular endothelial growth factor A. J Biol Chem 276: 11323-11334.

Lobo MK, Karsten SL, Gray M, Geschwind DH, Yang XW (2006). FACS-array profiling of striatal projection neuron subtypes in juvenile and adult mouse brains. Nat Neurosci 9: 443-452.

Lobo MK, Zaman S, Damez-Werno DM, Koo JW, Bagot RC, DiNieri JA et al (2013). DeltaFosB induction in striatal medium spiny neuron subtypes in response to chronic pharmacological, emotional, and optogenetic stimuli. J Neurosci 33: 18381-18395.

Maze I, Chaudhury D, Dietz DM, Von Schimmelmann M, Kennedy PJ, Lobo MK et al (2014). G9a influences neuronal subtype specification in striatum. Nat Neurosci 17: 533-539.

Maze I, Covington HE 3rd, Dietz DM, LaPlant Q, Renthal W, Russo SJ et al (2010). Essential role of the histone methyltransferase G9a in cocaine-induced plasticity. Science 327: 213-216.

McClung CA, Nestler EJ (2003). Regulation of gene expression and cocaine reward by CREB and DeltaFosB. Nat Neurosci 6: 1208-1215.

Mitchell AC, Javidfar B, Pothula V, Ibi D, Shen EY, Peter CJ et al (2017). MEF2C transcription factor is associated with the genetic and epigenetic risk architecture of schizophrenia and improves cognition in mice. Mol Psychiatry (e-pub ahead of print).

Nestler EJ (2008). Review. Transcriptional mechanisms of addiction: role of DeltaFosB. Philos Trans R Soc Lond Ser B 363: 3245-3255.

Neve RL, Neve KA, Nestler EJ, Carlezon WA Jr (2005). Use of herpes virus amplicon vectors to study brain disorders. BioTechniques 39: 381-391.

Pena CJ, Bagot RC, Labonte B, Nestler EJ (2014). Epigenetic signaling in psychiatric disorders. J Mol Biol 426: 3389-3412.

Robison AJ, Nestler EJ (2011). Transcriptional and epigenetic mechanisms of addiction. Nat Rev Neurosci 12: 623-637.

Robison AJ, Vialou V, Mazei-Robison M, Feng J, Kourrich S, Collins $\mathrm{M}$ et al (2013). Behavioral and structural responses to chronic cocaine require a feedforward loop involving DeltaFosB and calcium/calmodulin-dependent protein kinase II in the nucleus accumbens shell. J Neurosci 33: 4295-4307.

Robison AJ, Vialou V, Sun HS, Labonte B, Golden SA, Dias C et al (2014). Fluoxetine epigenetically alters the CaMKIIalpha promoter in nucleus accumbens to regulate DeltaFosB binding and antidepressant effects. Neuropsychopharmacology 39: 1178-1186.

Rotem A, Ram O, Shoresh N, Sperling RA, Goren A, Weitz DA et al (2015). Single-cell ChIP-seq reveals cell subpopulations defined by chromatin state. Nat Biotechnol 33: 1165-1172.
Schaefer A, Sampath SC, Intrator A, Min A, Gertler TS, Surmeier DJ et al (2009). Control of cognition and adaptive behavior by the GLP/G9a epigenetic suppressor complex. Neuron 64: 678-691.

Shetreat ME, Lin L, Wong AC, Rayport S (1996). Visualization of D1 dopamine receptors on living nucleus accumbens neurons and their colocalization with D2 receptors. J Neurochem 66: 1475-1482.

Snowden AW, Gregory PD, Case CC, Pabo CO (2002). Genespecific targeting of $\mathrm{H} 3 \mathrm{~K} 9$ methylation is sufficient for initiating repression in vivo. Curr Biol 12: 2159-2166.

Sun H, Kennedy PJ, Nestler EJ (2013). Epigenetics of the depressed brain: role of histone acetylation and methylation. Neuropsychopharmacology 38: 124-137.

Surmeier DJ, Ding J, Day M, Wang Z, Shen W (2007). D1 and D2 dopamine-receptor modulation of striatal glutamatergic signaling in striatal medium spiny neurons. Trends Neurosci 30: 228-235.

Surmeier DJ, Song WJ, Yan Z (1996). Coordinated expression of dopamine receptors in neostriatal medium spiny neurons. J Neurosci 16: 6579-6591.

Vialou V, Maze I, Renthal W, LaPlant QC, Watts EL, Mouzon E et al (2010a). Serum response factor promotes resilience to chronic social stress through the induction of DeltaFosB. J Neurosci 30: 14585-14592.

Vialou V, Robison AJ, Laplant QC, Covington HE 3rd, Dietz DM, Ohnishi YN et al (2010b). DeltaFosB in brain reward circuits mediates resilience to stress and antidepressant responses. Nat Neurosci 13: 745-752.

von Schimmelmann M, Feinberg PA, Sullivan JM, Ku SM, Badimon A, Duff MK et al (2016). Polycomb repressive complex 2 (PRC2) silences genes responsible for neurodegeneration. Nat Neurosc 19: 1321-1330.

Walsh JJ, Friedman AK, Sun H, Heller EA, Ku SM, Juarez B et al (2014). Stress and CRF gate neural activation of BDNF in the mesolimbic reward pathway. Nat Neurosci 17: 27-29.

Yung KK, Bolam JP, Smith AD, Hersch SM, Ciliax BJ, Levey AI (1995). Immunocytochemical localization of D1 and D2 dopamine receptors in the basal ganglia of the rat: light and electron microscopy. Neuroscience 65: 709-730.

Zachariou V, Bolanos CA, Selley DE, Theobald D, Cassidy MP, Kelz MB et al (2006). An essential role for DeltaFosB in the nucleus accumbens in morphine action. Nat Neurosci 9: 205-211.

Supplementary Information accompanies the paper on the Neuropsychopharmacology website (http://www.nature.com/npp)

Neuropsychopharmacology 